\title{
Infecciones producto de catéter venoso central y factores asociados en neonatos del Hospital José Carrasco Arteaga
}

Central Venous Catheter Infections and Associated Factors in Neonates at the José Carrasco Arteaga Hospital Infecções por Cateter Venoso Central e Fatores Associados em Neonatos do Hospital José Carrasco Arteaga

José Domínguez Ortega

joal_3456@outlook.com

https://orcid.org/0000-0001-6196-3087

Rebeca Sarango Vivanco

drarebecasarango@gmail.com

https://orcid.org/0000-0002-1195-0971
Kimberly Sandoya Maza

drakimsandoya@gmail.com

https://orcid.org/0000-0001-5660-6915

\section{Zoila Katherine Salazar Torres}

zsalazart@ucacue.edu.ec

https://orcid.org/0000-0002-7663-8049

\section{Universidad Católica de Cuenca, Azuay-Ecuador}

Recibido 10 de septiembre 2021 / Arbitrado y aceptado 27 de octubre 2021 / Publicado 30 de diciembre 2021

\section{RESUMEN}

La utilización de dispositivos intravasculares centrales para la administración de diversas sustancias los ha convertido en una herramienta esencial dentro del área de neonatología, sin embargo, su uso ha llevado al desarrollo de complicaciones infecciosas las cuales son cada vez más frecuentes. Objetivo. Determinar la prevalencia de infecciones producto de catéter venoso central y sus factores asociados en neonatos del Hospital José Carrasco Arteaga durante los años 2013 a 2018 . Materiales y métodos. Es un estudio cuantitativo analítico de cohorte longitudinal retrospectivo. La población estuvo conformada por las historias clínicas de neonatos a los cuales se les coloco catéter venoso central, según datos obtenidos del departamento de estadística del Hospital "José Carrasco Arteaga". El tamaño de la muestra fue calculado por un muestreo simple aleatorizado mediante el programa Epidat v4.2, con un nivel de confianza del 95\%, se obtuvo un tamaño de muestra de 172 neonatos. Para el análisis se utilizó estadística descriptiva. Resultados. Se determina una prevalencia de 56 neonatos con infección producto del uso de catéter venoso central equivalente al $32.6 \%$, predominando como agente etiológico causal el Estafilococo epidermidis; durante la realización del estudio, la prematuridad, el bajo peso al nacer y el tiempo de uso de catéter por más de 15 días son los factores que más se asocian con la infección. Conclusión. Se demostró que el bajo peso al nacer, neonatos pretérmino y el uso de catéter por más de 15 días son uno de los factores predisponentes.

Palabras clave: Infección; Catéter venoso central; Prevalencia; Factores de riesgo

\begin{abstract}
The use of central intravascular devices for the administration of various substances has made them an essential tool within the area of neonatology, however, their use has led to the development of infectious complications which are increasingly frequent. Objective. To determine the prevalence of infections caused by the central venous catheter and its associated factors in neonates of the José Carrasco Arteaga Hospital during the years 2013 to 2018 . Materials and methods. It is a quantitative analytical retrospective longitudinal cohort study. The population was made up of all neonates to whom a central venous catheter was placed, according to data obtained from the statistics department of the "José Carrasco Arteaga" Hospital. The sample size was calculated by a simple randomized sampling using the Epidat v4.2 program, with a confidence level of $95 \%$, a sample size of 172 neonates was obtained. Descriptive statistics were used for the analysis. Results. A prevalence of 56 neonates with infection resulting from the use of a central venous catheter was determined, equivalent to $32.6 \%$, with Staphylococcus epidermidis as the causal etiological agent; During the study, prematurity, low birth weight and time of catheter use for more than 15 days are the factors most associated with infection. Conclusion. Low birth weight, preterm infants and the use of a catheter for more than 15 days were shown to be one of the predisposing factors.
\end{abstract}

Key words: Infection; Central venous catheter; Prevalence; Risk factors 


\section{RESUMO}

O uso de dispositivos intravasculares centrais para a administração de diversas substâncias tem tornado-os uma ferramenta essencial dentro da área de neonatologia, porém, seu uso tem levado ao desenvolvimento de complicações infecciosas cada vez mais frequentes. Objetivo. Determinar a prevalência de infecções causadas pelo cateter venoso central e seus fatores associados em neonatos do Hospital José Carrasco Arteaga durante os anos de 2013 a 2018. Materiais e métodos. Trata-se de um estudo quantitativo analítico de coorte longitudinal retrospectivo. A população foi constituída por todos os neonatos aos quais foi colocado cateter venoso central, conforme dados obtidos no setor de estatística do Hospital "José Carrasco Arteaga". O tamanho da amostra foi calculado por uma amostragem aleatória simples no programa Epidat v4.2, com nível de confiança de $95 \%$, obteve-se um tamanho de amostra de 172 neonatos. Estatísticas descritivas foram utilizadas para a análise. Resultados. Foi determinada uma prevalência de 56 neonatos com infecção decorrente do uso de cateter venoso central, equivalente a $32,6 \%$, sendo o Staphylococcus epidermidis o agente etiológico causal; durante o estudo, prematuridade, baixo peso ao nascer e tempo de uso do cateter por mais de 15 dias são os fatores mais associados à infecção. Conclusão. Baixo peso ao nascer, prematuridade e uso de cateter por mais de 15 dias mostraram-se um dos fatores predisponentes.

Palavras-Chave: Infecção; Cateter venoso central; Prevalência; Fatores de risco

\section{INTRODUCCIÓN}

El catéter venoso central, o vía venosa central es un dispositivo el cual permite acceder al flujo sanguíneo de la vena cava superior por medio de la vena yugular interna, safena, vena subclavia y vena femoral (1). La utilización de dispositivos intravasculares centrales para la administración de terapia antibiótica parenteral, nutrición parenteral total, o como instrumento para la monitorización cardiovascular o hemodinámica, los han convertido en una herramienta esencial dentro del área de neonatología $(2,3)$.

La infección resulta una de las complicaciones más comunes de la colocación de un catéter venoso central, además de presentar frecuentemente una elevada morbilidad y una potencial letalidad asociada a esta complicación (4). Según Lona et al, la bacteriemia posterior a la colación de un catéter venoso central constituye la principal causa de infección nosocomial atribuible a estos dispositivos, conjuntamente presentando una mortalidad del $25 \%$ (5).

Actualmente la información que confieren Morocho et al., (2) en su estudio establece que la prevalencia de infecciones con cultivos positivos es del $41.8 \%(3)$ de todas las causas de infección intrahospitalaria en la unidad de neonatología, el $28 \%$ de estas se atribuyen a un catéter venoso central, además atribuye como principales agentes causales al Estafilococo Epidermidis (37.2\%), Klebsiella Pneumoniae $(17,9 \%)$ y Escherichia Coli $(13,55 \%)$.

Uno de los primeros factores a considerar es el sexo del neonato, a pesar de que no existe evidencia científica de la influencia del sexo sobre la prevalencia de la infección, se establece una mayor prevalencia en el sexo masculino (6).

Así mismo, un estudio indica que la edad gestacional es un factor importante dentro de la patogenia de la infección (7), la escaza madurez del sistema inmune en el recién nacido lo hace susceptible a adquirir infecciones con mayor facilidad, esto ocurre especialmente en los neonatos pretérmino, pues el limitado desarrollo de inmunidad y la falta de transferencia de anticuerpos maternos lo constituyen un factor importante de morbimortalidad asociada a infección (8).

Las causas atribuibles al problema pueden relacionarse tanto con el neonato o 
con el catéter utilizado, según Freeman (9) las infecciones asociadas a catéter venoso central tienen una relación muy elevada con características del paciente como el peso al nacer, el Apgar o prematurez; de entre todas destaca el peso al nacer, pues un paciente con un peso menor de 1500 gr tiene el doble de riesgo de padecer alguna complicación relacionada al uso de este dispositivo. Bentlin, en su estudio relaciona a un mayor tiempo de utilización de catéter con un mayor riesgo de desarrollar infección producto del catéter, asimismo de desarrollar sepsis neonatal (10).

En la actualidad tanto la decisión que tipo de catéter usar, sea de acceso central directo o periférico, dependerá del material del que será fabricado el catéter, lo cual es un factor que influye en la prevalencia de infecciones; se describe que el polietileno es el material al cual se adhieren los microorganismos con mayor frecuencia (2).

El tiempo de utilización de catéter venoso central representa un factor significativo para el paciente y se asocia a una manipulación constante por parte del personal de salud, por lo tanto, el riesgo aumenta mientras más días se mantenga el catéter; Sanderson et al., (11) describe que el riesgo aumenta a partir del sexto día de utilización, pero este mantiene un riesgo elevado durante las 2 semanas posteriores a la colocación de la vía central. En contraste un estudio realizado por Vergara et al., (12) determina que el porcentaje de infecciones en relación al tiempo que han usado el catéter de manera más sistemática, siendo un $2.9 \%$ en un lapso menor a 7 días,
$6.4 \%$ en un lapso de tiempo entre los 7 a 14 días, $10.4 \%$ en un lapso de 14 a 28 días y $14.3 \%$ en los que superaban los 28 días de uso.

En cuanto al sitio de inserción de la vía central, Freeman et al., (13) establece que los sitio más comúnmente elegidos para la inserción del catéter y que presentaban mayor índice de infecciones (67.8\%) eran las venas safena y femoral.

Mientras en otro estudio realizado por Cruz et al., (14) describen que la infección presenta mayor incidencia en la vena yugular derecha (24.6\%), esto lo asocian a diversos factores como la dificultad de colocación, dificultad de curación del sitio de inserción y el ser un área de mayor sudoración.

El objetivo por el cual se colocó el catéter central tiene importancia especial, Hammarskjöld et al., (4) indica que el riesgo de infección es más alto cuando el uso del catéter es para hemodiálisis, mientras que el riesgo disminuye sustancialmente cuando se administra antibióticos. Vergara, (12) concluye en su estudio que pacientes que reciben nutrición parenteral mediante vía central tienen siete veces más de padecer una infección producto del mismo. Otro factor que se determina es el número de punciones a venas periféricas que se realiza mientras se posee un catéter central, el cual incrementa el riesgo en $16 \%$ por cada punción venosa que se realiza (15).

La principal complicación asociada es la sepsis neonatal $(9,10)$, la misma se desarrolla debido a una bacteriemia a causa de la infección prevalente, esta destaca como 
complicación principal debido a su elevada morbilidad y mortalidad, en especial en prematuros y en niños con bajo peso al nacer representando una tasa de mortalidad de 1-5 por cada 1000 nacidos vivos (16).

Actualmente, el disponer de dispositivos de acceso venosos central en las unidades de neonatología en los hospitales implica un gran avance, pues nos permite controlar de un modo adecuado varios aspectos de la terapéutica durante tiempos más prolongados, además de que resulta una gran ayuda para realizar exámenes complementarios. Sin embargo, este procedimiento puede presentar varias complicaciones, de las cuales destacan las infecciones asociadas a catéter venoso central, pues constituyen una de las principales causas de infección en la unidad de cuidados intensivos neonatales (17).

\section{MATERIALES Y MÉTODOS}

Se trata de un estudio cuantitativo, analítico de corte transversal, de tipo retrospectivo, que abarcó de manera aleatoria todas las historias clínicas de los neonatos que hayan requerido el uso se catéter venoso central durante los años 2013-2018, en los cuales se han realizado 315 procedimientos en neonatos. El tamaño de muestra fue calculado por muestreo simple aleatorizado mediante el programa "Epidat v4.2". con una probabilidad de ocurrencia del $41.8 \%$, utilizando un porcentaje del $5 \%$ de error y un $95 \%$ de margen de confianza, obteniéndose una muestra de 172 neonatos tomados de forma aleatoria. La población cumplió con el criterio de inclusión establecido: Neonatos los cuales hayan necesitado de la colocación y utilización de catéter venoso central durante los años 2013-2018. Estos datos fueron obtenidos del departamento de estadística de un Hospital Ecuatoriano de tercer nivel.

Para la recolección de la información, se contó con un formulario de recolección de datos donde se registraron la información obtenida de las historias clínicas (características demográficas y clínicas) la cual fue digitada en hoja de Excel versión 2014. Una vez consolidada la información se utilizaron los programas estadísticos para un análisis cuantitativo más exhaustivo: PSPP versión 1.0.1 en español; con el que se construyeron las principales estadísticas descriptivas, obteniendo los principales gráficos y tablas de frecuencias para cada una de las variables del estudio. Las variables se describieron con base a medidas de tendencia central y de dispersión, además de distribuciones porcentuales.

Previo a la realización de la investigación, se solicitó aprobación del tema de estudio al departamento de docencia e investigación del Hospital Ecuatoriano de tercer nivel, con el permiso correspondiente para la accesibilidad al departamento de estadística para la revisión de historias clínicas. La investigación fue presentada y aprobada en sus diferentes etapas por el comité de Bioética. 


\section{RESULTADOS Y DISCUSIÓN}

De los 172 neonatos analizados el 57\% eran de sexo masculino, mientras que el $43 \%$ restante corresponde al sexo femenino; en cuanto a la edad gestacional se registra una media de 36,6 semanas D.E. \pm 3.002 manteniendo mayor prevalencia recién nacidos a término con un $70.3 \%$, mientras que el peso al nacer se mantiene en $2649.93 \mathrm{gr}$ D.E. \pm 671.716 teniendo el normopeso una prevalecía del $68 \%$, tal como se muestra en la Tabla 1.

Tabla 1. Características demográfica y clínicas de los recién nacidos participantes del estudio.

\begin{tabular}{llcccc}
\hline & Masculino & $\begin{array}{c}\text { Frecuencia } \\
(\mathbf{n})\end{array}$ & $\begin{array}{c}\text { Porcentaje } \\
(\%)\end{array}$ & Media & $\begin{array}{c}\text { Desviación } \\
\text { estándar }\end{array}$ \\
\hline \multirow{3}{*}{ Sexo del recién nacido } & Femenino & 98 & 57 & & \\
& Total & 74 & 43 & & \\
& A termino & 172 & 100 & & 3.002 \\
Edad gestacional & Pretérmino & 121 & 70,3 & & \\
& Total & 51 & 29,7 & 36.68 & \\
Peso al nacer & Normopeso & 172 & 100 & & \\
& Bajo peso & 117 & 68 & & \\
& Bajo peso extremo & 16 & 22,7 & & \\
& Total & 172 & 9,3 & & \\
\hline
\end{tabular}

Referente al catéter venoso central, el tipo de catéter venoso central más utilizado es el catéter de acceso directo con un $51.2 \%$, difiriendo con el catéter venoso central de inserción periférica con $47.1 \%$, la media de días que se utiliza un catéter venoso central es de 12.42 días D.E. \pm 5.97 ; el sitio de mayor frecuencia de inserción de catéter venoso central es la vena yugular interna $(40,7 \%)$, también se describen otros sitios de inserción además de los investigados como son el supraclavicular, braquial y radial; en cuanto al uso del catéter venoso central, el $83.1 \%$ corresponde a la administración de múltiples sustancias, mientras que su uso únicamente para la administración de líquidos (7\%) o medicamentos (9.9\%) es limitado (Tabla 2). 
Tabla 1. Datos referentes al catéter venoso central colocado en neonatos.

\begin{tabular}{|c|c|c|c|}
\hline & & Frecuencia (n) & Porcentaje (\%) \\
\hline \multirow{5}{*}{$\begin{array}{c}\text { Tipo de catéter venoso } \\
\text { central }\end{array}$} & Catéter venoso central de acceso directo & 88 & 51,2 \\
\hline & Catéter venoso central de inserción periférico & 81 & 47,1 \\
\hline & Catéter umbilical & 3 & 1,7 \\
\hline & Total & 172 & 100 \\
\hline & 1 a 7 días & 40 & 23,3 \\
\hline \multirow{6}{*}{$\begin{array}{c}\text { Tiempo de utilización de } \\
\text { catéter venoso central } \\
\text { (media } 12.42 \text { días DE } \\
\pm 5.97 \text { ) }\end{array}$} & 7 a 14 días & 78 & 45,3 \\
\hline & 14 a 28 días & 51 & 29,7 \\
\hline & Más de 28 días & 3 & 1,7 \\
\hline & Total & 172 & 100 \\
\hline & Yugular interna & 70 & 40,7 \\
\hline & Safena & 49 & 28,5 \\
\hline \multirow{5}{*}{$\begin{array}{l}\text { Sitio de inserción de } \\
\text { catéter venoso central }\end{array}$} & Subclavia & 16 & 9,3 \\
\hline & Femoral & 2 & 1,2 \\
\hline & Otros & 35 & 20,3 \\
\hline & Total & 172 & 100 \\
\hline & Medicamentos & 17 & 9,9 \\
\hline \multirow{3}{*}{$\begin{array}{c}\text { Uso del catéter venoso } \\
\text { central }\end{array}$} & Líquidos & 12 & 7 \\
\hline & Administración de múltiples sustancias & 143 & 83,1 \\
\hline & Total & 172 & 100 \\
\hline
\end{tabular}

En lo que corresponde a la prevalencia de infección confirmada por cultivo como se muestra en la Tabla 3, de los 172 casos recolectados, 56 presentaron infección confirmada por <cultivos de punta de catéter positivos para un germen, dando una prevalencia de $32.6 \%$ a la infección de catéter venoso central en neonatos.

Tabla 3. Prevalencia de infección confirmada mediante cultivo de punta de catéter venoso central en neonatos.

\begin{tabular}{ccc}
\hline & Frecuencia & Porcentaje (\%) \\
\hline Si & 56 & 32,6 \\
No & 116 & 67,4 \\
\hline Total & $\mathbf{1 7 2}$ & $\mathbf{1 0 0}$ \\
\hline
\end{tabular}


En la Tabla 4 se presentan los resultados sobre microrganismo con más prevalencia en cultivos de punta de catéter venoso central fue el Estafilococo epidermidis (46.4\%) seguido del Estafilococo aureus (21.4\%) y el Enterococus faecalis $(10.7 \%)$, entre otros.

Tabla 4. Microrganismos prevalentes en cultivo de punta de catéter en la unidad de neonatología.

\begin{tabular}{lcc}
\hline \multicolumn{1}{c}{ Microrganismo encontrado } & Frecuencia & Porcentaje (\%) \\
\hline Estafilococos epidermidis & 26 & 46,4 \\
Estafilococos aureus & 12 & 21,4 \\
Otros Estafilococos & 1 & 1,8 \\
Enterococus faecalis & 6 & 10.7 \\
Klebsiella pneumoniae & 4 & 7,1 \\
Escherichia coli & 2 & 3,6 \\
Pseudomona aeruginosa & 3 & 5.4 \\
Otros & 2 & 3,6 \\
\hline Total & $\mathbf{5 6}$ & $\mathbf{1 0 0}$ \\
\hline
\end{tabular}

En referente al análisis entre factores asociados y la presencia de infección de catéter venoso central destacado en la Tabla 5 , respecto al peso al nacer, la presencia de infección fue más prevalente en neonatos cuyo peso era inferior a los $2499 \mathrm{gr}(58.9 \%$ ) y menos prevalente en neonatos con peso mayor a 2500 gr (41.1\%) la diferencia fue estadísticamente significativa ( $p=0.00$ ), lo que se demuestra que los recién nacidos con bajo peso al nacimiento tienen mayor probabilidad de tener infección asociada al CVC (OR 6.88; IC 95\%: 3.35-14.12). Referente a la edad gestacional la presencia de infección en recién nacido pretérmino (51.8\%) fue menor a los recién nacido a término (48.2\%), esta diferencia fue estadísticamente significativa $(p=0.000)$, demostrando que la los recién nacidos pretérmino tienen más probabilidad de padecer infección producto de catéter venoso central (OR 5.61 IC 95\%: 2.76-11.82). 
Tabla 5. Relación entre factores y presencia de infección en neonatos del Hospital José Carrasco Arteaga 2013-2018.

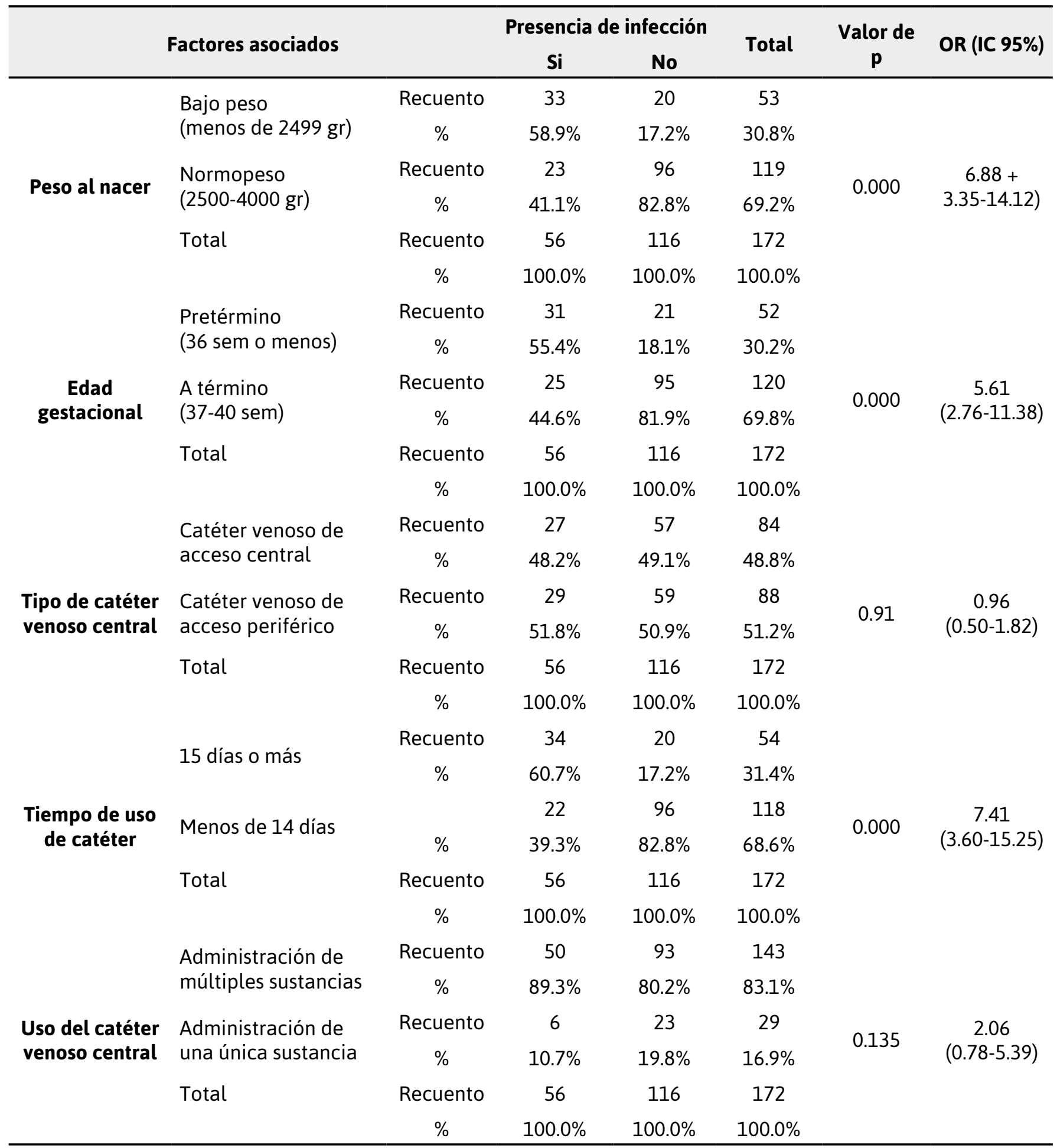


Con respecto al catéter venoso central como se muestra en la Tabla 5 , existe leve diferencia entre el catéter venoso central de acceso directo (48.2\%) y catéter venoso central de acceso periférico (51.8\%) siendo esta no estadísticamente significativa (0.91); mientras que, en el tiempo de uso de catéter venoso central, la infección fue más prevalente en catéter usados por más de 15 días (60.7\%) en comparación a catéteres usados por menos de 14 días (39.3\%). Esta diferencia es estadísticamente significativa; demostrando que el tiempo de uso de catéter durante 15 días o más, aumenta la probabilidad de padecer infecciones producto de catéter venoso central en neonatos (OR:7.41, IC95\%: 3.60-15.25). Mientras que, en relación al uso del catéter venoso central, para la administración de múltiples sustancias se muestran en mayor frecuencia (89.3\%) a la administración de sustancias únicas (10.7\%) siendo esta relación no estadísticamente significativa $(p=0.13)$.

\section{Discusión}

La utilización del catéter venoso central ha significado ungranavance para la supervivencia de neonatos en situación crítica, sin embargo, la prevalencia de infección que aún se asocia al uso de estos dispositivos demuestra un problema de salud aun presente (18). Según los resultados obtenidos del presente estudio, se establece que la prevalencia de infección de catéter venoso central en neonatos fue del $32.6 \%$, encontrándose un numero de variables asociadas a la infección, las cuales podrían determinar un rol importante en la aparición de esta patología.
Según Morocho y Torres, en un estudio realizado en el área de neonatología del Hospital Vicente Corral Moscoso establece una prevalencia infección de catéter del $41.8 \%$ (19), mientras que en un estudio realizado por Méndez, y Niola, determina que la prevalencia de infección de catéter es de 14.2\% (20); es así que se muestra una diferencia notoria entre la prevalencia que se mantiene entre 2 casas de salud de la ciudad de Cuenca, la cual se podría asociar a los protocolos que manejan las casas de salud; pese a ello, es evidente el aumento de la prevalencia que se mantiene en el trascurso de los años y la prevalencia en general durante los 5 años de estudio, lo cual nos lleva a analizar si los métodos de pesquisa han mejorado o si se incumplen los protocolos establecidos relacionados a técnica y tiempo de utilización.

Los neonatos analizados durante el estudio, fueron de predominio masculino (57\%), nacidos a término $(70.3 \%)$ y con un peso ubicado entre 2500 y $4000 \mathrm{gr}$ (68\%); datos que concuerdan con la información otorgada por el Ministerio de Salud Pública por medio del último informe anual del registro de nacimientos registrados en el sistema Revit del año 2017 (21).

La necesidad de conocer la etiología bacteriana de la infección radica no solo en las complicaciones infecciosas que se pueden presentar, sino también en la elección de un tratamiento antibiótico adecuado (22). En el estudio se observa una prevalencia marcada de cocos grampositivos, principalmente el Estafilococo epidermidis (46,4\%), teniendo menor porcentaje las bacterias nosocomiales, entre la que destaca la Klebsiella pneumoniae (7.1\%). 
Estos resultados son similares a los que nos brinda la literatura; Carter et al., (23) en su estudio describen como microorganismo predominante al Estafilococo epidermidis en las infecciones relacionadas con catéter venoso central; de igual modo, Concepción et al establecen como microorganismo predominante al Estafilococo coagulasa negativo (42.6\%) en infecciones relacionadas con dispositivos de uso médico (24). Se observa una marcada prevalencia de Estafilococo epidermidis como agente etiológico de infección asociada a catéter venoso central, tanto a nivel local como internacional; corroborando que estos microorganismos continúan siendo los más aislados en cultivos de Maki. El Estafilococo epidermidis demuestra que además de ser un comensal saprofito de la piel, que tiene un potencial patógeno elevado (25).

En cuantos a los factores asociados a la infección en el presente estudio se evaluó que el peso al nacer tiene una relación estadísticamente significativa, pues un peso menor a 2499 gr al nacer aumenta en 6,8 veces el riesgo de infección, contrastando, un estudio realizado por Morocho et al, describe un riesgo de 2.3 veces (26). Esta información se corrobora por Crivaro et al., (27) pues indica que mientras menos peso al nacer, más riesgo de contraer infección asociada a catéter.

Asimismo, un factor estudiado es la edad gestacional al nacimiento, pues este estudio determina que la prematuridad aumenta en 5.6 veces el riesgo de contraer infección asociada a CVC, Sánchez (28) establece que la prematurez aumenta en 1.6 veces el riesgo de contraer infección; mientras que, Cabrera et al., (29) quien determina que la prematuridad es un factor importante para considerar, pero en su estudio no consigue resultados estadísticamente significativos.

Otro factor importante por considerar es el tiempo de permanencia de catéter venoso central, en este estudio se registra una media de 12.42 días de utilización, mientras que, un estudio realizado por Morocho y Torres (26) establecen el tiempo de permanencia con media de 11.9 días; en contraste un estudio realizado por García et al., (30) México, determina una media de tiempo en 7 días. El tiempo de utilización por más de 15 días se asocian con riesgo de 7.41 veces contraer infección; además el tiempo de uso prolongado, predispone a más manipulación del catéter, y mayor probabilidad de colonización, lo cual aumenta considerablemente el riesgo de infecciones; información que se corrobora según la literatura, pues esta indica que existe un $25 \%$ de riesgo de infección a partir del décimo día de uso (31).

\section{CONCLUSIÓN}

En este estudio la prevalencia de la infección de catéter venoso central se muestra en un tercio de la población estudiada, siendo el microrganismo etiológico predominante el Estafilococo epidermidis.

Los factores asociados a infección de catéter venoso central fueron el bajo peso al nacer, neonatos pretérmino y el uso de catéter 
por más de 15 días. Es importante destacar que no se encontró relación significativa entre el tipo de catéter venoso central y el uso de este con la prevalencia de infección, por lo cual se sugiere continuar con protocolo de cultivo de punta de catéter cuando se realice el retiro de estos dispositivos independiente de la causa de extracción, pues de este modo se puede prevenir futuras complicaciones infecciosas en los neonatos.

\section{REFERENCIAS BIBLIOGRÁFICAS}

1. Chávez Ormaza GC, Cristina G. Implementación del protocolo de inserción y de manejo del catéter venoso central de larga permanencia totalmente implantable; estudio de los factores de riesgo y complicaciones, en los pacientes pediátricos, del Hospital Oncológico Solón Espinoza Ayala SO. Vol. 1. Universidad Internacional del Ecuador; 2017.

2.FerrerC,AlmiranteB.Infecciones relacionadas con el uso de los cateteres vasculares. Enferm Infecc Microbiol Clin. 2014;32(2):115-24.

3. Chen IL, Ou-Yang MC, Chen FS, Chung MY, Chen CC, Liu YC, et al. The equations of the inserted length of percutaneous central venous catheters on neonates in NICU. Pediatr Neonatol. 2018;1-6.

4. Hammarskjöld $F$, Berg $S$, Hanberger $H$, Taxbro K, Malmvall BE. Sustained low incidence of central venous catheter-related infections over six years in a Swedish hospital with an active central venous catheter team. Am J Infect Control. 2014;42(2):122-8.

5. Lona-Reyes JC, López-Barragán B, Rosa A de JC de la, Pérez-Molina JJ, Ascencio-Esparza EP. Sepsis neonatal temprana, incidencia y factores de riesgo asociados en un hospital público del occidente de México. Bol Méd Hosp Infant México. 2016;73(2):105-10.

6. María JJ, Rodríguez L, García S, Revilla
R. Utilización y mantenimiento de los catéteres venosos periféricos en la unidad de neonatología del hospital universitario río hortega, valladolid. Rev Enfermería Castilla Leon. 2015;7:3-11.

7. Rosado V, Camargos PAM, Anchieta LM, Bouzada MCF, Oliveira GM de, Clemente WT, et al. Fatores de risco para infecção associada a cateteres venosos centrais em população neonatal - revisão sistemática. J Pediatr (Rio J). 2018;94(1):3-14.

8. Garaboa NA, Portal YS, Marquez Y. El recién nacido pretérmino con infección de inicio precoz Clinical and epidemiological characterization infants with early-onset infection of preterm. Rev Cienc Médicas Pinar Río. 2015;19(6):1014-27.

9. Triviño J. Complicaciones infecciosas asociadas con catéter venoso central. Estudio realizado en el área de unidad de cuidados intensivos neonatales del Hospital de Especialidades Guayaquil Dr. Abel Gibert Pontón, en el periodo de Enero a Diciembre del 2015 [Tesis de Grado]. [Guayaquil-Ecuador]: Universidad de Guayaquil; 2015.

10. Zambrano A. Vía venosa central y complicaciones en neonatos hospitalizados en Francisco Icaza Bustamante 2014 - 2017 [Tesis de Grado]. [Guayaquil-Ecuador]: Universidad de Guayaquil; 2017.

11. Sanderson E, Yeo KT, Wang AY, Callander I, Bajuk B, Bolisetty S, et al. Dwell time and risk of central-line-associated bloodstream infection in neonates. J Hosp Infect. 2017;97(3):267-74.

12. Vergara T, Véliz E FA. Los días de exposición a nutrición parenteral aumentan el riesgo de bacteriemia asociada a catéter venoso central. Rev Chil Infectol. 2016;33(6):603-8.

13. Freeman JJ, Gadepalli SK, Siddiqui SM, Jarboe MD, Hirschl RB. Improving central line infection rates in the neonatal intensive care unit: Effect of hospital location, site of insertion, and implementation of catheterassociated bloodstream infection protocols. J Pediatr Surg. 2015;50(5):860-3. 
14. Cruz P, Rincon J, Mendieta G. Factores de riesgo asociados a infección de catéter venoso central. Investig Materno Infant. 2015;7(3):10715.

15. Cheng HY, Lu CY, Huang LM, Lee PI, Chen JM, Chang LY. Increased frequency of peripheral venipunctures raises the risk of central-line associated bloodstream infection in neonates with peripherally inserted central venous catheters. J Microbiol Immunol Infect. 2016;49(2):230-6.

16.LópezA, García M.Efectividad del uso delcare bundles en neonatología para la prevención de infecciones asociadas a la inserción del catéter venoso central de inserción periférica (PICC) [Tesis de Especialidad]. [Lima. Peru]: Universidad de Norbert Wiener; 2018.

17. Gil Gallego C, Lara Ruiz G, Hospital Universitario San Vicente Fundación, Cardona Arango D, Universidad CES. Demographic factors, technical and associated features bacteremia in patients with central venous catheter in intensive care unit HUSVF, 20092011. Salud Uninorte. 15 de noviembre de 2016;32(3):483-99.

18. Araújo FL de, Manzo BF, Costa ACL, Corrêa A dos R, Marcatto J de O, Simão DA da S. Adesão ao bundle de inserção de cateter venoso central em unidades neonatais e pediátricas. Rev Esc Enferm USP. 27 de noviembre de 2017;51(0).

19. Morocho F, Torres J. Perfil bacteriológico, susceptibilidad antibiótica y factores asociados al uso de los dispositivos intravasculares centrales de la Unidad de Neonatología del Hospital Vicente Corral Moscoso. Cuenca. 2017 [Tesis de Grado]. [Cuenca-Ecuador]: Universidad de Cuenca; 2018.

20. Mendez F, Niola F. Microrganismos frecuentes y resistencia antimicrobiana en muestras biológicas del area de neonatología de Hospital Vicente Corral Moscoso [Tesis de pregrado]. [Cuenca]: Universidad de Cuenca; 2015.
21. Arana T. Registro de datos vitales: Revit. Ministerio de salud pública; 2017 p. 8. (Registro de estadísticas vitales en linea-revit 2017).

22. Resende DS, Peppe ALG, dos Reis $H$, Abdallah VOS, Ribas RM, Gontijo Filho PP. Late onset sepsis in newborn babies: epidemiology and effect of a bundle to prevent central line associated bloodstream infections in the neonatal intensive care unit. Braz J Infect Dis. enero de 2015;19(1):52-7.

23. Carter $\mathrm{JH}$, Langley JM, Kuhle $\mathrm{S}$, Kirkland S. Risk Factors for Central Venous CatheterAssociated Bloodstream Infection in Pediatric Patients: A Cohort Study. Infect Control Hosp Epidemiol. agosto de 2016;37(8):939-45.

24. Concepción YM, Portal YS, Portal ME, Garaboa NA, Campos AC. Caracterización clínico-epidemiológica del recién nacido con infección asociada a los cuidados. Rev Cienc Medicas. 2015;19(6):1028-44.

25. González $M$, López $M$, Montesino $M$. Resistencia microbiana de microorganismos aislados en neonatología Hospital "Abel Santamaría "2015. Rev Cienc MEdicas Pinar Río. 2016;20(5):10.

26. Morocho F, Torres J. Perfil bacteriológico, susceptibilidad antibiótica y los factores asociados al uso de dispositivos intravasculares centrales de la unidad de neonatología del Hospital Vicente Corral Moscoso. Cuenca. 2017. Universidad de Cuenca; 2014.

27. Crivaro V, Bogdanovi L, Bagattini M, lula VD, Catania M, Raimondi F, et al. Surveillance of healthcare-associated infections in a neonatal intensive care unit in Italy during 2006-2010. BMC Infect Dis. diciembre de 2015;15(1):152.

28. Sánchez R. Factores de riesgo relacionados con infecciones del torrente sanguíneo en neonatos con catéter venoso central en unidad de cuidados intensivos Hospital Regional del IHSS, San Pedro Sula, Cortés, Honduras, julio 2011 a junio 2012 [Tesis de Posgrado]. [Honduras]: Universidad Nacional Autónoma de Honduras; 2014. 
29. Cabrera D, Cuba F. Incidencia de las infecciones del torrente sanguíneo asociadas a catéter central y factores de riesgo relacionados a su ocurrencia en una unidad de cuidados intensivos neonatales de un hospital público de nivel III-1. [Tesis de Posgrado]. [Perú]: Universidad Cayetano Heredia; 2019.

30. García H, Torres-Gutiérrez J, PeregrinoBejarano L. Factores de riesgo asociados a infección nosocomial (IN) en una Unidad de Cuidados Intensivos Neonatales (UCIN) de tercer nivel. Gac Médica México. 2015;151(7):9.
31. Villamarín-Bello $B$, Piñeiro-Lamas $M$, Barros-Dios JM, Ruano-Ravina A, García-Otero MJ, Fernández-Villanueva JR. Bacteremia nosocomial asociada a catéter vascular central en unidades de cuidados intensivos en 2 hospitales en Galicia (España). Infection. abril de 2016;20(2):62-9.

Conflicto de intereses: Ninguno declarado por los autores.

Financiación: Ninguna declarada por los autores.

Agradecimiento: Ninguno manifestado por los autores

\section{ACERCA DE LOS AUTORES}

José Domínguez Ortega. Médico General, Escuela de medicina de la Universidad católica de Cuenca. Experiencia en funciones hospitalarias y administrativas, capacitación médica, atención en todos los grupos etarios, Diagnóstico y tratamiento de diferentes patologías. Universidad Católica de Cuenca, Ecuador.

Kimberly Sandoya Maza. Médica General, Universidad Católica de Cuenca, Ecuador. Experiencia en diagnósticos, elaboración de estrategias de tratamiento óptimos adecuados a la condición de los pacientes y cuidados preventivos. Universidad Católica de Cuenca, Ecuador.

Rebeca Sarango Vivanco. Médica General, Universidad Católica de Cuenca, Ecuador. Experiencia en funciones hospitalarias, atención médica integral, Diagnóstico y tratamientos de calidad de acuerdo con el paciente. Universidad Católica de Cuenca, Ecuador.

Zoila Katherine Salazar Torres. Médico especialista en Ginecología y Obstetricia. Magister en Investigación de la Salud. Universidad Católica de Cuenca, Ecuador. 